\title{
Cisplatin-Induced Posterior Reversible Encephalopathy Syndrome-Brief Report and Review of the Literature
}

\author{
Limor Amit $^{1,2}$, Irit Ben-Aharon ${ }^{1,2 \#}$, Thomas Tichler ${ }^{1,2}$, Edna Inbar $^{2,3}$, Aaron Sulkes ${ }^{1,2}$, \\ Salomon M. Stemmer ${ }^{1,2}$ \\ ${ }^{1}$ Institute of Oncology, Davidoff Center, Rabin Medical Center, Beilinson Campus, Petah-Tiqva, Israel \\ ${ }^{2}$ The Sackler Faculty of Medicine, Tel Aviv University, Tel Aviv, Israel \\ ${ }^{3}$ Department of Radiology, Rabin Medical Center, Beilinson Campus, Petah-Tiqva, Israel \\ Email: "iritbenaharon@gmail.com
}

Received July 13, 2011; revised August 28, 2011; accepted October 13, 2011

\begin{abstract}
Cisplatin is one of the most broadly used chemotherapeutic agents. Several central nervous system toxicities have been attributed to this agent, among which is a rare clinicoradiological condition referred to as posterior reversible leukoencephalopathy syndrome (PRES) or reversible posterior leukoencephalopathy syndrome (RPLS). PRES is characterized by subacute onset of headache, visual disturbances, confusion, seizures and rarely, coma. This usually reversible syndrome was associated in the past mainly with hypertension. Several reports have associated the syndrome with several immunosuppressive and chemotherapeutic agents suggesting a disruption of the blood brain barrier due to direct damage to the endothelium and vasogenic edema. This syndrome is readily diagnosed with Computed Tomography (CT) or Magnetic resonance imaging (MRI) studies and quick withdrawal of the causative agent in conjunction with supportive care including rapid hypertension control and reduction of elevated intracranial pressure when needed, usually reverse the neurological symptoms with minimal or even no sequela within several days. In this brief report and review of the literature we describe two cases of PRES in two female patients that occurred a few days after receiving Cisplatin-based chemotherapy. We discuss the clinical presentation, diagnosis and treatment of this syndrome by reviewing the literature. The escalating number of clinical reports of PRES merit further studies of the mechanism of toxicity, appropriate treatment and awareness of physicians to this life threatening entity
\end{abstract}

Keywords: PRES; RPLS; Encephalopathy; Cisplatin; Hypertension

\section{Introduction}

Cisplatin, a platinum compound, is one of the most broadly used chemotherapeutic agents. Since its introduction in the 1970s Cisplatin has become a cornerstone for many of the anti-cancer regimens in a variety of malignancies. Peripheral nerves that are exposed to the highest levels of Cisplatin are at greatest risk, due to deposit of the metal metabolites, while central structures are usually more protected from the accumulation of these metabolites [1], although several case reports have been published regarding the central nervous system neurotoxicity of Cisplatin.

We herein describe two cases and a review of the literature, of Cisplatin effects on the central nervous system (CNS), reflected mainly as PRES.

\section{Case Presentation}

\subsection{Patient \#1}

A 26 year old female with no significant medical history,

\footnotetext{
* Conflict of interest statement: The authors declare no conflicts of interests.

${ }^{\#}$ Corresponding author.
}

presented with carcinosarcoma of the peritoneum, she was treated with combination chemotherapy including: Doxorubicin, Ifosfamide, Mesna and Cisplatin, followed by granulocyte colony stimulating factor (G-CSF) support on days 5 - 10 of the cycle. Twelve days after her third cycle of chemotherapy the patient presented with seizures (5 generalized seizures within 30 hours) and hypertension (up to $180 \mathrm{mmHg}$ systolic and $110 \mathrm{mmHg}$ diastolic pressure) that was treated with Clonidine $0.15 \mathrm{mg} \times 2 / \mathrm{d}$, (an $\alpha$-blocker due to acute renal failure and benzodiazepines for the seizures). Her physical examination revealed no neurological deficit at first but deteriorated two days later, when the patient had generalized seizures, and hypo-reflexia of the lower extremities. Laboratory evaluation was notable for severe metabolic acidosis, a creatinine level of $4.7 \mathrm{mg} / \mathrm{dl}$ and thrombocytopenia of 32,000 per $\mu \mathrm{l}$ (microliter), bilirubin was slightly elevated to a value of 2.1 $\mathrm{mg} / \mathrm{dl}$ with mild cholestatic enzyme elevation. Hemoglobin, white blood cell count and electrolytes were within normal limits.

A lumbar puncture was preformed and revealed mildly 
elevated protein and glucose levels in the cerebrospinal fluid (CSF), and no cells were identified. Bacterial cultures and serological tests for herpes virus and West Nile Virus were negative. In the following MRI study there was a diffuse symmetrical signal abnormality in the grey and white matter of both cerebral hemispheres but more dominant in the occipital and parietal lobes (Figure 1(a)). These findings were consistent with PRES. Due to worsening metabolic acidosis, worsening pancytopenia and fever, the patient was intubated and transferred to the intensive care unit, where she received supportive care consisting of mannitol $40 \mathrm{gr} \times 4 / \mathrm{d}$, antibiotics, G-CSF and anti-hypertensive medications (Amlodipine $5 \mathrm{mg} \times 1 / \mathrm{d}$ and then diltiazem $60 \mathrm{mg} \times 3 / \mathrm{d}$ ). Urinary output was adequate during the entire episode. Within 3 days the patient regained consciousness and was extubated. CT, performed 6 weeks later showed remarkable improvement of the edema (Figure 1(b)). One month later the patient was released with a "drop foot" as the only neurological sequel. She was not able to receive further treatment due to her low tolerance. Seven months later the patient died of respiratory failure due to progression of her underlying disease.

\subsection{Patient \#2}

A 61 year old female with no significant medical history, was diagnosed with metastatic gastric carcinoma to the liver, presented with neutropenic fever and abdominal pain 7 days after her first course of chemotherapy that included Docetaxel, Cisplatin and 5-Fluorouracil (5FU). Her physical examination revealed abdominal tenderness and her laboratory findings were notable for neutropenia and direct hyperbilirubinemia. The patient was treated with antibiotics and G-CSF with resolution of her symptoms.

Five days later she developed confusion and focal seizures that progressed into generalized seizures and right hemiparesis concomitant with sudden onset of hypertension (systolic increased from $100-120 \mathrm{mmHg}$ to 140 $180 \mathrm{mmHg}$ and diastolic increased from $70-80 \mathrm{mmHg}$ to $90-110 \mathrm{mmHg}$ ), urinary output was normal. CT with contrast was remarkable for marked bilateral occipital edema consistent with PRES (Figure 2(a)). The patient was intubated due to loss of consciousness and a Glasgow coma scale score of 5 and was transferred to the intensive care unit where she received supportive care with Dexamethasone $20 \mathrm{mg} \times 1 / \mathrm{d}$ and mannitol $40 \mathrm{~g} \times 4 / \mathrm{d}$ in an effort to reduce the elevated intracranial pressure and anti-hypertensive medications: after an initial dose of IV labetalol 20mg the patient was changed to Prolol $10 \mathrm{mg} \times$ $3 / \mathrm{d}$ with Disothiazide $25 \mathrm{mg} \times 1 / \mathrm{d}$. The patient regained consciousness and was extubated four days later, with no neurological sequela. In the follow up MRI six weeks later there was only minimal signal abnormality adjacent to the atrium of the left lateral ventricle (Figure 2(b)).

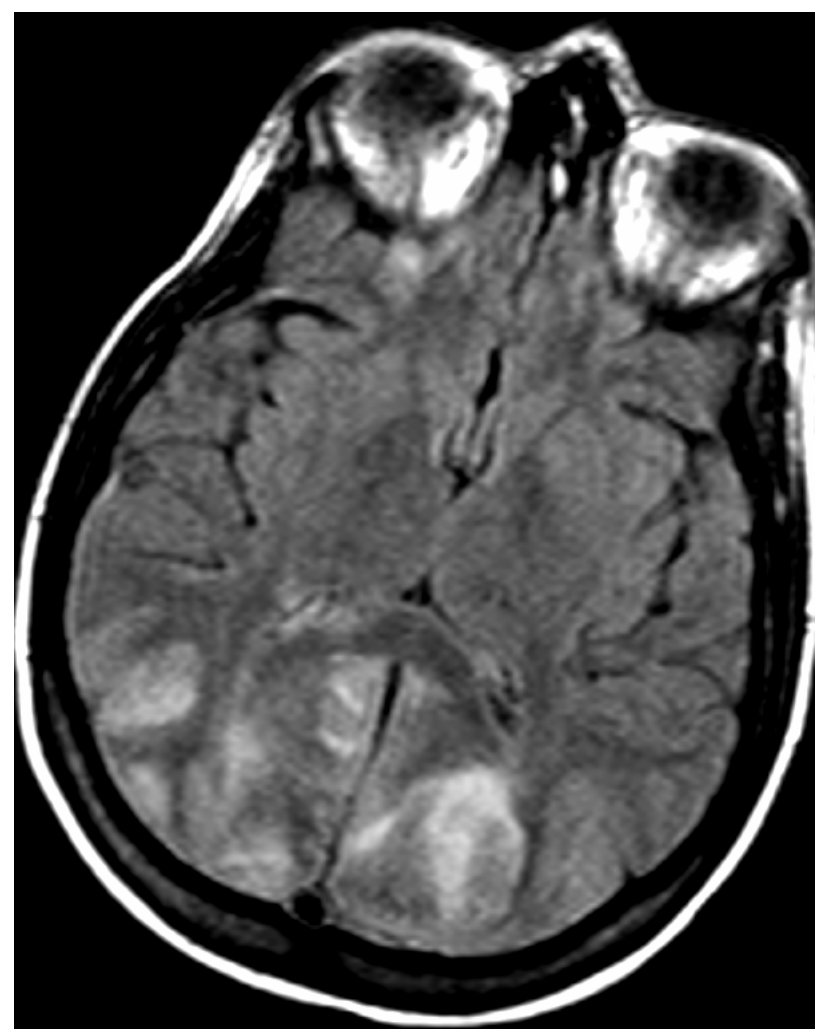

(a)

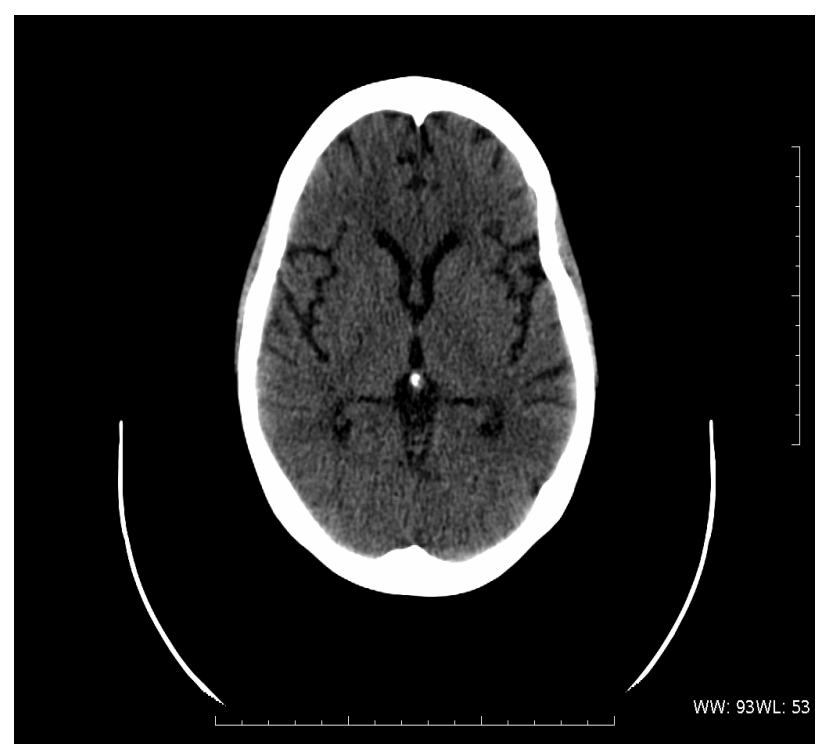

(b)

Figure 1. A 26-year-old woman presented with seizures, hypertension and hyporeflexia following cisplatin treatment. (a) Cranial MRI obtained 2 days after the onset of symptoms revealed typical features of PRES include bilateral, symmetric hyperintensities involving the posterior cerebral white matter; (b) Follow-up CT image obtained after 6 weeks shows resolution of the lesions. 


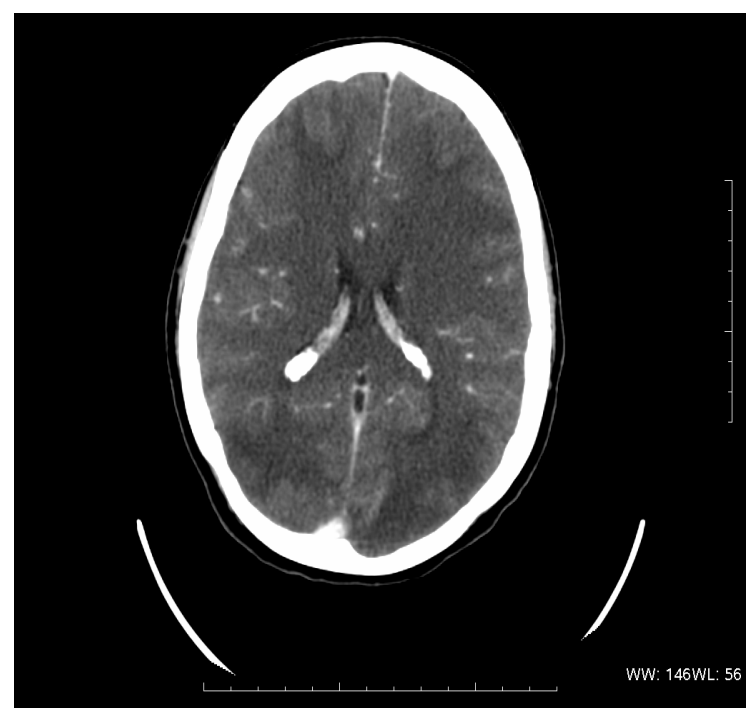

(a)

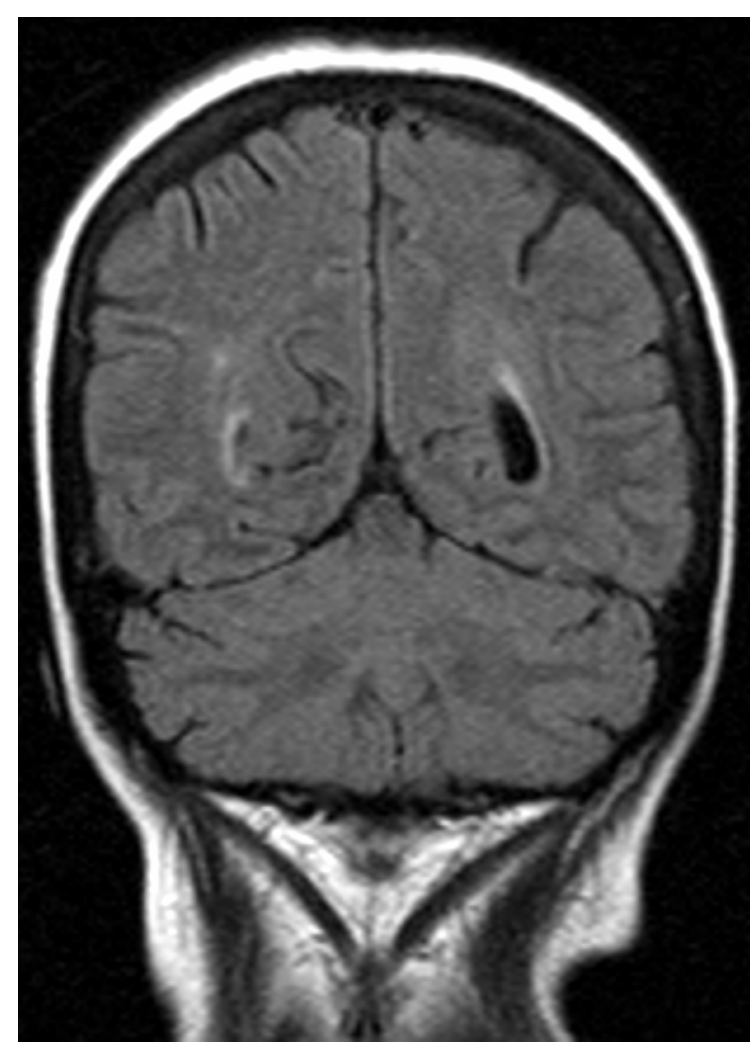

(b)

Figure 2. A 61-year-old woman presented with encephalopathy, seizures and right hemiparesis following cisplatin treatment. (a) CT obtained at the onset of symptoms reveals typical features of PRES; (b) Follow-up cranial MRI obtained after 6 weeks shows resolution of the lesions.

\section{Review of the Literature}

PRES or RPLS, both refer to a clinicoradiological entity characterized by a subacute onset of headache, confusion, seizures, and visual disturbances, manifesting typically with transient changes in the posterior circulation territory of the brain [1-4]. Upon physical examination, tendon reflexes are often brisk and some patients experience weakness and incoordination of the limbs [1].

The radiological appearance depicts a pattern of edema which usually affects the subcortical white matter of the parietooccipital region in a symmetrical manner. In classic cases, brain MRI demonstrates symmetric T2 hyperintensities involving the parietal and occipital white matter bilaterally [5]. The only published pathological case report of a brain biopsy in a patient diagnosed with PRES, described white matter edema without other recognizable abnormalities [6]. Though known as "reversible", PRES is neither persistently reversible nor always localized exclusively to the posterior territory of the brain. In many cases, gray matter is involved in addition to white matter [7]. A retrospective study assessed the reversibility of PRES due to various etiologies and found that the typical cortical and subcortical PRES lesions were usually reversible, whereas the brain stem and deep white matter lesions showed less reversibility [4]. Casey et al. [2] proposed that the term PRES should be changed to RPLS, to stress the common involvement of both the grey and the white matter.

In most of the PRES cases the phenomenon correlates with malignant hypertension, eclampsia or hypertension secondary to renal diseases. Though formerly PRES has been attributed mostly to abrupt and severe hypertensive encephalopathy, several reports have described the syndrome with little or no hypertension [8].

While the mechanism of hypertensive PRES has been proposed to be a breakdown of cerebral blood pressure and hence disruption of the auto-regulation of cerebral vessels, the mechanism by which immunosuppressive and cytotoxic agents cause PRES has been proposed to be related to the alteration of the blood-brain barrier due to direct impairment of the endothelium [4,9-11]. This results in extravasation of fluid into the extracellular space and vasogenic edema. The posterior circulation may be more susceptible due to a relative lack of the sympathetic innervation, and therefore is potentially more susceptible to hypertension. Of note, G-CSF has been correlated to the presence of PRES as well $[1,8]$. The latter may be more common than reported as several reports of patients with PRES presumably induced by chemotherapy were simultaneously treated with G-CSF. However due to the considerable numbers of patients that receive G-CSF routinely and the paucity of reports, it is highly doubtful that this agent causes the PRES phenomenon; nonetheless, synergism between G-CSF and cytotoxic agents can not be excluded.

PRES is a rare yet documented complication of chemotherapy and immunosuppressive agents such as Cytosine Arabinoside, Cyclosporine, and Tacrolimus [3,7]. 
Several case reports in the literature acknowledged PRES as a neurological adverse effect of Cisplatin [12-19]. There are two neurological patterns of Cisplatin induced CNS toxicity. The first consists of focal neurological deficits or decreased level of consciousness, with or without seizures. Symptoms may develop a few hours and up to few months after exposure to the agent, and are not dose dependent. No radiological pattern is evident in these cases, and CSF analysis is normal. Electroencephalography (EEG) shows a generalized slowing pattern (sometimes with epileptic discharges). Symptoms usually resolve without permanent neurological deficits. Other Cisplatin-induced toxicities such as neutropenia, hypomagnesemia, hyponatremia, hypokalemia and renal dysfunction are described as contributory factors [20]. The second pattern consists of PRES, with the characteristic symptoms as described above and the typical neuroradiological abnormalities. This occurs usually immediately at the end or shortly after (within few days) Cisplatin administration, though as described in our first case report, the syndrome may occur after several dosages of Cisplatin as well. Despite the severe clinical symptoms and signs, most patients make a full recovery. Some authors stated a possible interaction between Cisplatin and general anesthetic agents, or associated electrolyte or blood pressure disturbances that precipitate the occurrence of PRES [21].

It has also been reported that Cisplatin-induced PRES can be reflected as mild encephalopathy without seizures, yet with the typical radiological pattern [22].

Of note, a case of PRES has been reported in a young patient with metastatic adenocarcinoma of the rectum who received Oxaliplatin, a platinum compound as well, who developed seizures and altered mental status and a typical pattern of PRES on MRI [23]. Carboplatin-induced PRES has also been reported recently [24].

Hypomagnesemia often accompanies Cisplatin-induced PRES. Considering that magnesium sulphate is used to treat eclamptic convulsions (some of which have PRES traits), magnesium might be involved in the pathophysiology of PRES and physicians should cautiously observe the magnesium levels of these patients [25].

There are neither guidelines for supportive treatment of PRES nor evidence for the best type of therapy. Since the majority of the reported cases were resolved with the use of intravenous antihypertensive medications and magnesium sulphate, these are widely used in the management of this syndrome. In order to determine the magnitude of this syndrome, a special registry should be considered for better estimation and future tailoring of treatment guidelines for this life threatening situation. In most case reports of PRES due to various etiologies, a female predominance has been depicted. This observation, may imply (based on the known association to preeclampsia), a role for host mechanisms such as autoimmunity, which is more evident in women. Future studies should explore this aspect as well.

The rising number of reports of this condition due to different chemotherapy and immunosuppressive agents such as Gemcitabine, Cyclosporine, biological therapies like Bevacizumab and Sunitinib $[8,26,27]$ and agents like G-CSF [24] should raise the threshold of suspicion in physicians and neuroradiologists treating this group of patients [28].

\section{Conclusions}

Cisplatin-induced PRES should be suspected in patients who have recently received Cisplatin and develop neurological symptoms. Imaging of the brain reveals a typical leukoencephalopathy pattern which is usually more pronounced in the posterior territory of the brain due to anatomical differences in its innervation. The importance of this report lies in the acute, life threatening but reversible nature of this condition when recognized and treated promptly by withdrawal of the suspected offending drug, swift control of both arterial hypertension and raised intracranial pressure and respiratory support when necessary.

The escalating number of clinical reports of PRES merits further studies of the mechanism of toxicity, adequate treatment and awareness of physicians to this life threatening entity.

\section{REFERENCES}

[1] J. Hinchey, C. Chavez, B. Appignani, J. Breen, L. Pao, A. Wang, et al., "A Reversible Posterior Leukoencephalopathy Syndrome," The New England Journal of Medicine, Vol. 334, No. 8, 1996, pp. 494-500. doi:10.1056/NEJM199602223340803

[2] S. O. Casey, R. C. Sampaio, E. Michel and C. L. Truwit, "Posterior Reversible Encephalopathy Syndrome: Utility of Fluid-Attenuated Inversion Recovery MR Imaging in the Detection of Cortical and Subcortical Lesions," American Journal of Neuroradiology, Vol. 21, No. 7, 2000, pp. 1199-1206.

[3] C. Lamy, C. Oppenheim, J. F. Meder and J. L. Mas, "Neuroimaging in Posterior Reversible Encephalopathy Syndrome," Journal of Neuroimaging, Vol. 4, No. 2, 2004, pp. 89-96.

[4] A. R. Pande, K. Ando, R. Ishikura, Y. Nagami, Y. Takada, A. Wada, et al., "Clinicoradiological Factors Influencing the Reversibility of Posterior Reversible Encephalopathy Syndrome: A Multicenter Study," Radiation Medicine, Vol. 24, No. 10, 2006, pp. 659-668. doi:10.1007/s11604-006-0086-2

[5] T. Kinoshita, T. Moritani, D. A. hrier, A. Hiwatashi, H. Z. Wang, Y. Numaguchi, et al., "Diffusion Weighted MR Imaging of Posterior Reversible Leukoencephalopathy Syndrome: A Pictorial Essay," Clinical Imaging, Vol. 27, No. 5, 2003, pp. 307-315. 


\section{doi:10.1016/S0899-7071(02)00592-2}

[6] D. Schiff and M. B. Lopes, "Neuropathological Correlates of Reversible Posterior Leukoencephalopathy," Neurocritical Care, Vol. 2, No. 3, 2005, pp. 303-305.

doi:10.1385/NCC:2:3:303

[7] A. Norden and T. Batchelor, "Reversible Posterior Leukoencephalopathy Syndrome after Treatment of Diffuse Large B-Cell Lymphomam" Onkologie, Vol. 30, No. 3, 2007, pp. 90-91.

[8] A. Rajasekhar and T. J. George, "Gemcitabine-Induced Reversible Posterior Leukoencephalopathy Syndrome: A Case Report and Review of the Literature," The Oncologist, Vol. 12, No. 11, 2007, pp. 1332-1335

[9] J. De Seze, B. Mastain, T. Stojkovic, D. Ferriby, J. P. Pruvo, A. Destee, et al., "Unusual MR Findings of the Brain in Arterial Hypertension," American Journal of Neuroradiology, Vol. 21, No. 2, 2000, pp. 391-394.

[10] H. Kitaguchi, H. Tomimoto, Y. Miki, A. Yamamoto, K. Terada, H. Satoi, et al., "A Brainstem Variant of Reversible Posterior Leukoencephalopathy Syndrome," Neuroradiology, Vol. 47, No. 9, 2005, pp. 652-656. doi:10.1007/s00234-005-1399-Z

[11] T. Shimono, Y. Miki, H. Toyoda, H. Egawa, S. Uemoto, K. Tanaka, et al., "MR Imaging with Quantitative Diffusion Mapping of Tacrolimus-Induced Neurotoxicity in Organ Transplant Patients," European Radiology, Vol. 13, No. 5, 2003, pp. 986-993.

[12] I. J. Berman and M. P. Mann, "Seizures and Transient Cortical Blindness Associated with Cis-Platinum (II) Diamminedichloride (PDD) Therapy in a Thirty-Year-Old Man," Cancer, Vol. 45, No. 4, 1980, pp. 764-766. doi:10.1002/1097-0142(19800215)45:4<764::AID-CNCR 2820450425>3.0.CO;2-G

[13] M. T. Cattaneo, V. Filipazzi, E. Piazza, E. Damiani and G. Mancarella, "Transient Blindness and Seizure Associated with Cisplatin Therapy," Cancer Research and Clinical Oncology, Vol. 114, No. 5, 1988, pp. 528-530. doi:10.1007/BF00391507

[14] R. N. Hitchins and D. B. Thomson, "Encephalopathy Following Cisplatin, Bleomycin and Vinblastine Therapy for non-Seminomatous Germ Cell Tumour of Testis," Australian and New Zealand Journal of Medicine, Vol. 18, No. 1, 1988, pp. 67-68. doi:10.1111/j.1445-5994.1988.tb02244.x

[15] P. A. Philip, J. Carmichael and A. L. Harris, "Convulsions and transient Cortical Blindness after Cisplatin," British Medicine Journal, Vol. 302, No. 6773, 1991, p. 416. doi:10.1136/bmj.302.6773.416

[16] M. Highley, S. T. Meller and C. R. Pinkerton, "Seizures and Cortical Dysfunction Following High-Dose Cisplatin Administration in Children," Medical and Pediatric Oncology, Vol. 20, 1992, pp. 143-148. doi: $10.1002 / \mathrm{mpo} .2950200210$

[17] T. Van Gelder, P. Geurs, G. S. Kho, D. W. J. Dippel, Ch. J. Vecht, T. A. W. Splinter, "Cortical Blindness and Sei- zures Following Cisplatin Treatment: Both of Epileptic Origin?" European Journal of Cancer, Vol. 29, No. 10, 1993, pp. 1497-1498. doi:10.1016/0959-8049(93)90030-J

[18] D. C. Young, A. Mitchell, J. Kessler and J. E. Christman, "Cortical Blindness and Seizures Possibly Related to Cisplatin, Vinblastine and Bleomycin Treatment of Ovarian Dysgerminoma," The Journal of the American Osteopathic Association, Vol. 93, No. 2, 1993, pp. 502-504.

[19] K. Nomura, R. Ohno, K. Hamaguchi, T. Hata, H. Hatanaka and H. Matsuyama, "Clinicopathological Report of Cisplatin Encephalopathy," Rinsho Shinkeigaku, Vol. 35, No. 1, 1995, pp. 64-69.

[20] N. Steeghs, F. E. de Jongh, P. A. S. Smitt and M. J. van den Bent, "Cisplatin-Induced Encephalopathy and Seizures," Anti-Cancer Drugs, Vol. 14, No. 6, 2003, pp. 443446. doi:10.1097/00001813-200307000-00009

[21] P. S. Rangi, W. J. Partridge, E. S. Newlands and A. D. Waldman, "Posterior Reversible Encephalopathy Syndrome: A Possible Late Interaction between Cytotoxic Agents and General Anaesthesia," Neuroradiology, Vol. 47, No. 8, 2005, pp. 586-590. doi:10.1007/s00234-005-1376-6

[22] O. Lyass, A. Lossos, A. Hubert, M. Gips and T. Peretz, "Cisplatin-Induced Non-Convulsive Encephalopathy," AntiCancer Drugs, Vol. 9, No. 1, 1998, pp. 100-104. doi:10.1097/00001813-199801000-00013

[23] D. M. Pinedo, F. Shah-Khan and P. C. Shah, "Reversible Posterior Leukoencephalopathy Syndrome Associated with Oxaliplatin," Journal of Clinical Oncology, Vol. 25, No. 33, 2007, pp. 5320-5321. doi:10.1200/JCO.2007.13.5954

[24] S. Vieillot, D. Pouessel, N. M. de Champfleur and C. Becht, S. Culine, "Reversible Posterior Leukoencephalopathy Syndrome after Carboplatin Therapy," Annals of Oncology, Vol. 18, No. 3, 2007, pp. 608-609.

[25] Y Ito, Y Arahata, Y Goto, M Hirayama, M Nagamutsu, T Yasuda, et al., "Cisplatin Neurotoxicity Presenting as Reversible Posterior Leukoencephalopathy Syndrome," American Journal of Neuroradiology, Vol. 19, No. 3, 1998, pp. 415-417.

[26] M. D. Haefner, "Reversible Posterior Leukoencephalopathy Syndrome after Treatment of Diffuse Large B-Cell Lymphoma," Onkologie, Vol. 30, No. 3, 2007, pp. 90-91. doi:10.1159/000098706

[27] G. Martín, L. Bellido and J. J. Cruz, "Reversible Posterior Leukoencephalopathy Syndrome Induced by Sunitinib," Journal of Clinical Oncology, Vol. 25, No. 23, 2007, p. 3559.

[28] C. Ozcan, S. J. Wong and P. Hari, "Reversible Posterior Leucoencephalopathy Syndrome and Bevacizumab," The New England Journal of Medicine, Vol. 354, No. 9, 2006, pp. 980-982. doi:10.1056/NEJMc052954

[29] R. M. Connolly, "Chemotherapy Induced Reversible Posterior Leukoencephalopathy Syndrome," Lung Cancer, Vol. 56, No. 3, 2007, pp. 459-463. doi:10.1016/j.lungcan.2007.01.012 